\title{
Optimización en la asignación de tareas en un sistema de guardería forestal*
}

Optimal assignment program for forest rangers positioning and procedure

\author{
LORENA PRADENAS ROJAS ${ }^{1}$, LEANDRO AZOCAR GARCIA ${ }^{2}$
}

${ }^{1}$ Departamento de Ingeniería Industrial, Universidad de Concepción, Casilla 160-C, Concepción, Chile, Email: 1pradena@udec.cl

${ }^{2}$ Magíster en Ingeniería Industrial, Universidad de Concepción y Forestal Arauco, email: lazocar@arauco.cl

\section{SUMMARY}

We solved the forest ranger assignment problem (FRAP) by using the algorithm of the nearest location in the salesman traveling problem (STP). The FRAP consists of assigning tasks to a system of surveillance and forest administration. The solution proposal is to apply a solution to the problem that optimizes the tasks of forest rangers supervising 27.000 ha of forest with 102 sectors, and 402 nodes. The algorithm is evaluated twice a year, winter and summer. There are two initial solution forms (1) To roam forest rangers that leave the home node randomly or (2) to go to the nearest location. Neither solution differs significantly, either in cost or total distance traveled. The forest rangers can be scheduling with $99 \%$ accuracy, thus creating a considerable improvement over the already existing one.

Key words: harvest assignment forest, traveling salesman problem, combinatorial optimization.

\section{RESUMEN}

En este artículo se propone el uso de la heurística del vecino más cercano, que resuelve el problema del vendedor viajero (PVV), para optimizar la asignación de tareas en un sistema de vigilancia y gestión forestal (PAGF). La solución propuesta es aplicada a la vigilancia de un patrimonio de 27.000 ha, conteniendo 102 predios y 402 nodos. El algoritmo es evaluado en dos épocas del año, invierno y verano, considerando que las salidas de los guardias desde el nodo de pernoctación es: (1) de manera aleatoria o (2) hacia el vecino más cercano. Las soluciones alcanzadas son similares en costo y distancia total, la programación de las actividades de los guardabosques alcanzada es con una certeza del 99\%, proporcionando así una mejora considerable en la planificación de dichas tareas. Los tiempos computacionales obtenidos también son satisfactorios.

Palabras clave: asignación de guardias forestales, problema del vendedor viajero, optimización combinatoria.

\section{INTRODUCCION}

El sector forestal chileno ha experimentado un fuerte crecimiento en las últimas décadas, lo que se ha traducido en un incremento muy significativo en la cantidad de plantaciones, razón por la cual las empresas forestales han aumentado considerablemente la cantidad de predios bajo su administración.
En este contexto, la administración de estos predios resulta compleja, debido principalmente a la amplia distribución geográfica y a riesgos de daños por parte de terceros. Entonces, es interesante sistematizar la vigilancia y el control de las tareas requeridas en los predios bajo administración y disminuir, así, los riesgos mencionados.

La empresa Bosques Arauco S.A. administra un patrimonio forestal superior a 250.000 ha, las

\footnotetext{
* Con apoyo parcial de Proyecto Fundación Andes C-13955/18 y Proyecto UDEC 204.097.007-1.0
} 
que se encuentran divididas en ocho áreas administrativas: Santa Juana, Laraquete, Arauco, Curanilahue, Lebu, Cañete, Angol y Collipulli, presentando superficies promedio de 30.000 hectáreas, 110 predios y 6 guardabosques cada una.

En el presente estudio se realiza una propuesta de solución para la asignación de guardias forestales, la cual es aplicada al área de Lebu; el modelo es utilizado en una red de 402 nodos. En la figura 1 se entrega un esquema de la situación a modelar.

La solución es analizada, según un modelo del problema del vendedor viajero (TSP), que es uno de los problemas más estudiados dentro de la optimización combinatorial; se trata de un problema fácil de enunciar, pero difícil de resolver, sobre todo cuando existen situaciones de gran tamaño, donde sólo es posible tratar con algoritmos heurísticos.

El Problema del vendedor viajero TSP $(1,2)$ consiste en que un vendedor, que se encuentra en la ciudad origen, debe visitar exactamente una vez un conjunto de ciudades vecinas de tal forma de obtener una ruta de costo mínimo, comenzando y finalizando en la ciudad origen.

El Problema de $M$ vendedores viajeros (1) corresponde a una generalización del problema del vendedor viajero, donde se dispone de más de un vendedor. Los $M$ vendedores salen de un de- pósito común y regresan al mismo punto. Cada vehículo debe visitar por lo menos un nodo. El objetivo es minimizar la distancia total viajada por los $\mathrm{M}$ vendedores. Cada vendedor debe viajar a través de un subconjunto de nodos, que incluye un depósito común, y cada nodo (a excepción del depósito) debe ser visitado exactamente una sola vez y por un solo vendedor.

El Problema de programación y ruteo de vehículos $(3,4,5)$ considera un conjunto de tareas (o viajes) caracterizados por un origen, un destino, una duración y un tiempo de inicio fijo. Las tareas están dadas a priori y compuestas de una o varias actividades. Entre dos tareas sucesivas existe un período de tiempo de tránsito, con duración y costo. El problema a resolver consiste en formar un programa y secuencias de tareas, donde cada tarea es desarrollada exactamente una vez. Estas secuencias son algunas veces denominadas rutas.

Muchos problemas en la administración de distribución de productos o servicios pueden ser formulados como un problema de ruteo de vehículos (VRP, "vehicle routing problem", un ejemplo práctico se encuentra en, (6)). El objetivo del VRP es atender a un conjunto de clientes con una demanda por servicio a un costo mínimo a través de rutas que se originan y terminan en un depósito central. El VRP típico considera una flo-

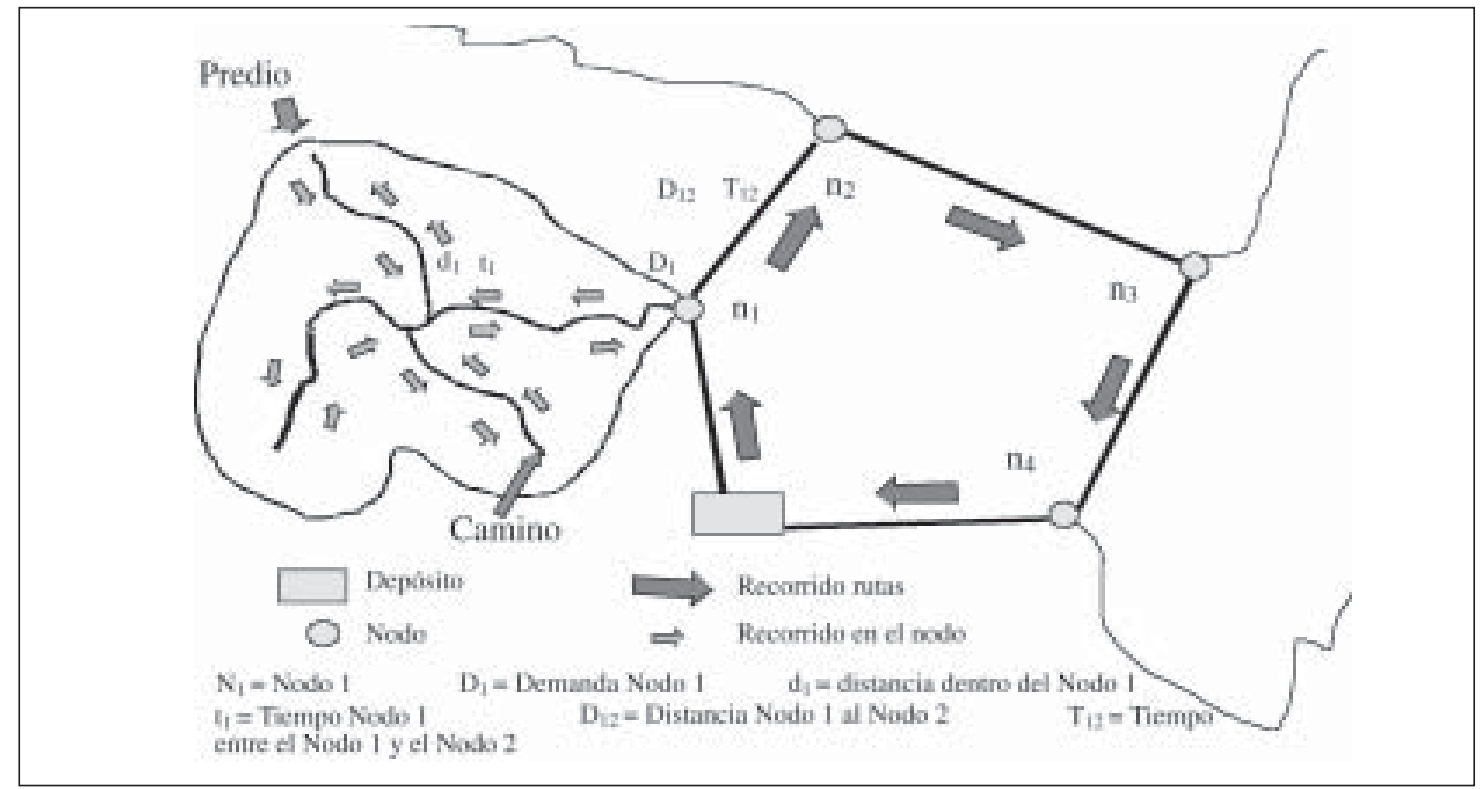

Figura 1. Descripción general de la red de vigilancia.

General description of the network. 
ta de vehículos ubicados en un depósito central que pueden ser programados para proveer algún tipo de servicio a clientes geográficamente dispersos en una región a atender.

Los problemas tipo VRP consideran el diseño de rutas de atención (distribución o recolección) desde uno o más depósitos centrales a un conjunto de clientes dispersos. Varias versiones del problema pueden ser definidas, dependiendo del número de factores, restricciones y objetivos; así se dispone de situaciones donde:

- El problema puede ser de distribución, recolección o ambos.

- La distribución se lleva a cabo desde un depósito o varios.

- El número de vehículos considerados puede ser fijo o variable.

- La flota de vehículos puede ser homogénea o heterogénea.

- Condición de trabajo de los conductores.

- La demanda es determinística o estocástica.

- Los clientes ser atendidos dentro de una ventana de tiempo.

- Etc.

Las restricciones del VRP se pueden clasificar con relación a:

\section{VEHICULOS}

- Cada vehículo tiene un límite de capacidad.

- Cada vehículo tiene un tiempo total de trabajo desde su partida en el depósito hasta su regreso, generalmente condicionado por las horas de trabajo.

- Cada vehículo tiene un tiempo dentro del cual debe dejar el depósito y así asegurar espacio para otros vehículos de regreso al depósito.

- Cada vehículo tiene un costo asociado a la distribución o recolección del servicio entregado.

\section{CLIENTES}

- Cada cliente tiene una demanda que debe ser satisfecha. En algunos casos esta cantidad es conocida exactamente (Determinística) y en otros casos se conoce con cierto grado de incertidumbre (Estocástica, 8, 9).

- Cada cliente tiene un período de tiempo durante el cual la atención debe ocurrir (time windows).
- Cada cliente tiene un tiempo asociado de atención o visita.

- Cada cliente tiene un número de vehículos que no lo pueden atender (restricción de acceso).

- Cada cliente puede tener una prioridad de atención (condición climática).

- Cada cliente puede aceptar visitas divididas (más de un vehículo).

\section{OTROS FACTORES}

- Viajes múltiples del mismo vehículo en un día, el vehículo retorna al depósito y sale nuevamente.

- Más de un depósito.

Objetivos

- Minimizar el número de vehículos utilizados (costos fijos).

- Minimizar la distancia total (tiempo) resolución. (costos variables).

- Minimizar alguna combinación de las dos primeras (costos totales).

- Maximizar la función de utilidad basada en el servicio o conveniencia.

- Maximizar la función de utilidad basada en la prioridad de los clientes

Una de las características recurrentes de los problemas de optimización combinatorial es el hecho de que son muy "fáciles" de entender y de enunciar, pero generalmente son difíciles de resolver. En la actualidad, la investigación se ha dirigido hacia el diseño de buenas heurísticas, es decir, algoritmos eficientes con respecto al tiempo computacional y al espacio de memoria; y con cierta verosimilitud de entregar una solución "buena", esto es, relativamente cercana a la óptima mediante el examen de sólo un pequeño subconjunto de soluciones del espacio de solución.

Debido a la alta complejidad computacional que demanda resolver un problema de determinación de rutas en forma exacta, se han creado procedimientos que resuelven los problemas en un menor tiempo, aunque esto signifique que la solución obtenida sea aproximada. Estos procedimientos de solución reciben el nombre de heurísticas. Entre sus ventajas destacan que proporcionan soluciones a grandes problemas, con un costo computacional menor. 
BOSQUE 26(2): 17-24, 2005

Optimización en la asignación de tareas en un sistema de guardería forestal

\section{SOLUCION PROPUESTA}

Las heurísticas de solución para el problema del vendedor viajero las podemos clasificar en tres clases: procedimientos de construcción de rutas, procedimiento de mejora de rutas y procedimientos compuestos. Dentro de las primeras podemos mencionar: la heurística del vecino más cercano, el algoritmo de Clark y Wright (7) y los procedimientos de inserción entre otros.

Dadas las características del problema tratado en este estudio, el método utilizado para la solución es la heurística del vecino más cercano, considerando que la primera salida del día es en forma aleatoria a cualquiera de los nodos disponibles para cada guardabosque.

El procedimiento del vecino más cercano contiene básicamente las siguientes etapas:

Paso 1. Comenzar con cualquier nodo en forma aleatoria como el comienzo de la ruta.

Paso 2. Encontrar el nodo más cercano al último nodo incluido en la ruta, unir este nodo a la ruta, si es que son respetadas las restricciones de tiempo disponible, si no regresar al punto 1 .

Paso 3. Repetir el paso 2 hasta que todos los nodos estén contenidos en una ruta o se haya completado el tiempo total disponible. El peor de los casos con esta heurística sucede cuando:

Largo de la ruta del vecino más cercano $\leq 1 / 2[\lg (n)]+1 / 2$

Largo de la ruta óptima

Donde $l g$ denota el logaritmo natural en base 2, y $n$ el número de nodos en la red.
Una de las variaciones introducidas en el algoritmo para tratar el problema planteado es generar una salida aleatoria en el nodo inicial, lo que, dada la naturaleza del problema, produce un factor sorpresa frente a la absoluta incertidumbre acerca del nodo en el cual se presenta el evento.

\section{RESULTADOS}

El algoritmo es evaluado considerando una salida inicial al nodo más cercano del nodo de pernoctación, y una salida aleatoria desde el nodo de pernoctación; además, se considera la aplicación durante la época de verano e invierno para el área en estudio (10).

Los resultados para una época de verano se entregan en el cuadro 1; en términos generales para la salida aleatoria se dispone de una mayor distancia resolución (2\%), un mayor costo total (3\%) y un cumplimiento inferior en un $3 \%$ de la demanda total.

Al analizar las componentes de la distancia total resolución por cada guardabosque (cuadro 2), se observa una sobrecarga de trabajo para el guardabosque 2, lo que indica una desigual asignación de predios para él. Por otro lado, la proporción de distancia resolución en viajes de traslados es homogénea, con la excepción de los guardabosques 3 y 1 con un $77 \%$ y $61 \%$ de la distancia total resolución en viajes de traslado, lo que indicaría un problema de ubicación física del nodo de pernoctación para estos.

\section{CUADRO 1}

Resultados época verano, distancias y tiempos totales.

Summer period results, distances and total times.

\begin{tabular}{|c|c|c|c|c|c|c|c|c|}
\hline \multirow[b]{2}{*}{ Guardabosque } & \multicolumn{4}{|c|}{ Salida aleatoria } & \multicolumn{4}{|c|}{ Salida al nodo más cercano } \\
\hline & $\begin{array}{c}\text { Distancia total } \\
\mathrm{km}\end{array}$ & $\begin{array}{c}\text { Tiempo total } \\
\mathrm{Hr}\end{array}$ & $\begin{array}{c}\text { Costo total } \\
\mathrm{M} \$\end{array}$ & $\begin{array}{l}\text { Núm. Visitas } \\
\text { Unidades }\end{array}$ & $\begin{array}{c}\text { Distancia total } \\
\mathrm{km}\end{array}$ & $\begin{array}{c}\text { Tiempo total } \\
\mathrm{Hr}\end{array}$ & $\begin{array}{c}\text { Costo total } \\
\mathrm{M} \$\end{array}$ & $\begin{array}{l}\text { Núm. Visitas } \\
\text { Unidades }\end{array}$ \\
\hline 1 & 1.001 & 98 & 224 & 185 & 1.057 & 107 & 236 & 203 \\
\hline 2 & 1.731 & 208 & 388 & 277 & 1.721 & 210 & 385 & 288 \\
\hline 3 & 643 & 44 & 144 & 72 & 551 & 41 & 123 & 72 \\
\hline 4 & 346 & 41 & 78 & 60 & 346 & 41 & 78 & 60 \\
\hline 5 & 930 & 107 & 208 & 143 & 880 & 105 & 197 & 143 \\
\hline 6 & 821 & 90 & 184 & 129 & 789 & 89 & 176 & 129 \\
\hline Total & 5.472 & 588 & 1.226 & 866 & 5,344 & 593 & 1.195 & 895 \\
\hline
\end{tabular}


BOSQUE 26(2): 17-24, 2005

Optimización en la asignación de tareas en un sistema de guardería forestal

\section{CUADRO 2}

Distribución de la distancia ocupada.

Coverage distribution.

\begin{tabular}{|cccccccc|}
\hline & \multicolumn{3}{c}{ Salida aleatoria } & & \multicolumn{3}{c|}{ Salida al nodo más cercano } \\
\cline { 2 - 3 } \cline { 7 - 8 } Guardabosque & $\begin{array}{c}\text { Distancia } \\
\text { viaje km }\end{array}$ & $\begin{array}{c}\text { Distancia } \\
\text { nodo } \mathrm{km}\end{array}$ & $\begin{array}{c}\text { Distancia } \\
\text { total km }\end{array}$ & $\begin{array}{l}\text { Distancia } \\
\text { viaje } \mathrm{km}\end{array}$ & $\begin{array}{c}\text { Distancia } \\
\text { nodo km }\end{array}$ & $\begin{array}{c}\text { Distancia } \\
\text { total km }\end{array}$ \\
\hline 1 & 612 & 389 & 1.001 & & 623 & 434 & 1.057 \\
2 & 848 & 883 & 1.731 & & 808 & 913 & 1.721 \\
3 & 494 & 149 & 643 & & 402 & 149 & 551 \\
4 & 175 & 171 & 346 & & 175 & 171 & 346 \\
5 & 474 & 456 & 930 & & 424 & 456 & 880 \\
6 & 437 & 384 & 821 & & 405 & 384 & 789 \\
Total & 3.040 & 2.432 & 5.472 & & 2.837 & 2.507 & 5.344 \\
\hline
\end{tabular}

Respecto del cumplimiento de la demanda (cuadro 3), para el caso de la salida aleatoria ésta se cumple en un $83 \%$, siendo de un $86 \%$ para la salida al nodo más cercano. El mayor incumplimiento de la demanda se evidencia en el guardabosque 2; como ya fue mencionada, la sobrecarga de trabajo para éste es el motivo de este incumplimiento, que para el caso de la salida aleatoria es de un $36 \%$ y para la salida al nodo más cercano es de un $33 \%$.

Los resultados para la época de invierno (cuadro 4) nos muestran que para la salida aleatoria se obtuvo una mayor distancia total resolución (6\%), un mayor costo total $(6 \%)$ y un cumplimiento menor $(1 \%)$ de la demanda total con relación a la salida al nodo más cercano.
Al analizar los componentes de la distancia total resolución (cuadro 5), encontramos que la distancia total resolución por el guardabosque 2 es muy superior a la de los demás, lo que confirma la necesidad de una revisión en la asignación de predios. La distancia de viaje es mayor en un $11 \%$ para la salida aleatoria con relación a la del nodo más cercano.

Para la salida aleatoria, la proporción entre distancia resolución de traslado y de recorrido propiamente tal dentro de cada nodo, la primera es mayor en un $26 \%$, es decir, se requieren más desplazamientos en llegar a los nodos que la distancia necesaria en su recorrido. Para la salida al nodo más cercano esta proporción baja a un $17 \%$, lo que remarca lo indicado anteriormente con re-

\section{CUADRO 3}

Demanda versus cumplimiento.

Demand versus fulfillment.

\begin{tabular}{|ccccccc|}
\hline \multirow{2}{*}{ Guardabosque } & $\begin{array}{c}\text { Demanda } \\
\text { unid. }\end{array}$ & $\begin{array}{c}\text { Visitas } \\
\text { unid. }\end{array}$ & $\begin{array}{c}\text { Cumplimienton } \\
\%\end{array}$ & & $\begin{array}{c}\text { Sisitas } \\
\text { unid. }\end{array}$ & $\begin{array}{c}\text { Cumplimiento } \\
\%\end{array}$ \\
\hline 1 & 203 & 185 & 91 & 203 & 100 \\
2 & 432 & 277 & 64 & & 288 & 67 \\
3 & 72 & 72 & 100 & & 72 & 100 \\
4 & 60 & 60 & 100 & & 60 & 100 \\
5 & 143 & 143 & 100 & & 143 & 100 \\
6 & 129 & 129 & 100 & & 129 & 100 \\
Total & 1.039 & 866 & 83 & 895 & 86 \\
\hline
\end{tabular}


BOSQUE 26(2): 17-24, 2005

Optimización en la asignación de tareas en un sistema de guardería forestal

\section{CUADRO 4}

Resultados distancia, tiempo y costos época de invierno.

Results of distance, time and winter period costs.

\begin{tabular}{|c|c|c|c|c|c|c|c|c|}
\hline \multirow[b]{2}{*}{ Guardabosque } & \multicolumn{4}{|c|}{ Salida aleatoria } & \multicolumn{4}{|c|}{ Salida al nodo más cercano } \\
\hline & $\begin{array}{l}\text { Distancia total } \\
\mathrm{km}\end{array}$ & $\begin{array}{c}\text { Tiempo total } \\
\mathrm{Hr}\end{array}$ & $\begin{array}{c}\text { Costo total } \\
\mathrm{M} \$\end{array}$ & $\begin{array}{l}\text { Núm. Visitas } \\
\text { Unidades }\end{array}$ & $\begin{array}{c}\text { Distancia total } \\
\mathrm{km}\end{array}$ & $\begin{array}{c}\text { Tiempo total } \\
\mathrm{Hr}\end{array}$ & $\begin{array}{c}\text { Costo total } \\
\mathrm{M} \$\end{array}$ & $\begin{array}{c}\text { Núm. Visitas } \\
\text { Unidades }\end{array}$ \\
\hline 1 & 431 & 41 & 97 & 96 & 416 & 40 & 93 & 96 \\
\hline 2 & 1.428 & 146 & 319 & 203 & 1.372 & 145 & 305 & 205 \\
\hline 3 & 444 & 29 & 99 & 50 & 375 & 27 & 84 & 50 \\
\hline 4 & 241 & 30 & 54 & 42 & 248 & 29 & 56 & 44 \\
\hline 5 & 625 & 68 & 140 & 98 & 581 & 67 & 130 & 98 \\
\hline 6 & 622 & 68 & 139 & 98 & 589 & 67 & 132 & 98 \\
\hline Total & 3.791 & 382 & 848 & 587 & 3.581 & 375 & 800 & 591 \\
\hline
\end{tabular}

\section{CUADRO 5}

Distribución de la distancia total para época de invierno.

Distribution of total distance for winter period.

\begin{tabular}{|c|c|c|c|c|c|c|}
\hline \multirow[b]{2}{*}{ Guardabosque } & \multicolumn{3}{|c|}{ Salida aleatoria } & \multicolumn{3}{|c|}{ Salida al nodo más cercano } \\
\hline & $\begin{array}{l}\text { Distancia } \\
\text { viaje } \mathrm{km}\end{array}$ & $\begin{array}{l}\text { Distancia } \\
\text { nodo } \mathrm{km}\end{array}$ & $\begin{array}{l}\text { Distancia } \\
\text { total } \mathrm{km}\end{array}$ & $\begin{array}{l}\text { Distancia } \\
\text { viaje } \mathrm{km}\end{array}$ & $\begin{array}{l}\text { Distancia } \\
\text { nodo } \mathrm{km}\end{array}$ & $\begin{array}{l}\text { Distancia } \\
\text { total } \mathrm{km}\end{array}$ \\
\hline 1 & 268 & 163 & 431 & 253 & 163 & 416 \\
\hline 2 & 799 & 629 & 1.428 & 728 & 634 & 1.372 \\
\hline 3 & 345 & 99 & 444 & 276 & 99 & 375 \\
\hline 4 & 117 & 124 & 241 & 119 & 129 & 248 \\
\hline 5 & 330 & 295 & 625 & 286 & 295 & 581 \\
\hline 6 & 324 & 298 & 622 & 291 & 298 & 589 \\
\hline Total & 2.183 & 1.608 & 3.791 & 1.953 & 1.618 & 3.581 \\
\hline
\end{tabular}

\section{CUADRO 6}

Cumplimiento demanda para época de invierno.

Fulfillment of demand for winter period.

\begin{tabular}{|ccccccc|}
\hline \multirow{2}{*}{ Guardabosque } & $\begin{array}{c}\text { Demanda } \\
\text { unid. }\end{array}$ & $\begin{array}{c}\text { Sisitas } \\
\text { unid. }\end{array}$ & $\begin{array}{c}\text { Cumplimiento } \\
\%\end{array}$ & & $\begin{array}{c}\text { Salida aleatoria } \\
\text { unid. }\end{array}$ & $\begin{array}{c}\text { Cumplimiento } \\
\%\end{array}$ \\
\hline 1 & 96 & 96 & 100 & & 96 & 100 \\
2 & 221 & 203 & 92 & & 205 & 93 \\
3 & 50 & 50 & 100 & & 50 & 100 \\
4 & 44 & 44 & 100 & & 44 & 100 \\
5 & 98 & 98 & 100 & & 98 & 100 \\
6 & 98 & 98 & 100 & & 98 & 100 \\
Total & 607 & 589 & 97 & & 591 & 97 \\
\hline
\end{tabular}


lación a la ubicación geográfica de los nodos de pernoctación de los guardabosques.

El análisis de la demanda para la época de invierno (cuadro 6) nos muestra que todos los guardabosques satisfacen la demanda en un $100 \%$, con excepción del guardabosque 2 en que se llega a un $92 \%$ de cumplimiento, para el caso de la salida aleatoria, y un $93 \%$ para la salida al nodo más cercano.

\section{CONCLUSIONES}

El algoritmo utilizado permite detectar que existen tiempos ociosos de algunos guardabosques que pueden ser corregidos mediante una mejor asignación de los predios bajo su responsabilidad. Esta situación queda reflejada tanto para la época de verano como la de invierno.

Los resultados obtenidos permiten concluir que el problema debe ser resuelto en forma integral, y no considerar a priori una asignación de predios a cada guardabosque, esta asignación debe ser realizada por el modelo o se debe mejorar considerablemente la asignación de predios a cada guardabosque, para homologar la carga de trabajo a cada uno de éstos.

Se hace indispensable la resolución con ventanas de tiempo para el período de verano, al requerir más de una visita en el día a algunos nodos con este tipo de demanda. Situación que no es contemplada en el problema del vendedor viajero.

La integración gráfica del problema permite una mayor fluidez al operador para la correcta asignación de los trabajos.

Para algunos guardabosques, se detecta que la ubicación de su nodo de pernoctación actual aumenta el tiempo de traslados, en desmedro de los tiempos de supervisión directa sobre los nodos. Lo que, de acuerdo a algún criterio económico, nos permite evaluar el cambio de ubicación física de aquel guardabosque en el que su costo de desplazamiento sea un porcentaje significativo del tiempo efectivamente trabajado.

En términos generales se obtienen mejores respuestas en costos, distancia total resolución y tiempos de recorrido, con la solución que sale desde el nodo de pernoctación al nodo más cercano, con relación a la que tiene una solución aleatoria. Con la particularidad que, dada la naturale- za del problema, la salida aleatoria, es impredecible para los agentes causantes de daños al patrimonio forestal administrado por cada guardabosque.

Por condiciones de demanda, la solución para la época de verano concentra una mayor distancia resolución, mayor costo y mayor tiempo de trabajo. Esto se fundamenta en que, durante la época de verano, aumentan los riesgos, dado que todo el patrimonio administrado se encuentra accesible.

Con relación a tiempos computacionales, no se observa diferencia entre la salida aleatoria y la del vecino más cercano. Sí se observa una diferencia importante entre las soluciones de verano e invierno, dada por la mayor demanda de atención en la época de verano.

$\mathrm{El}$ algoritmo propuesto entrega un plan diario de trabajo con una frecuencia preestablecida de verificación del patrimonio, lo que indudablemente produce una mejora sustancial al sistema actual utilizado en la empresa, que se basa en la experiencia del guardabosque, el cual define la ruta diaria a realizar.

Al proporcionar un plan diario de actividades, es factible dimensionar para el área de estudio la cantidad mínima de guardabosques requeridos para el trabajo, lo que permite en un futuro próximo realizar algún nivel de "outsourcing”, permitiendo una reducción considerable de los costos de administración.

Con los resultados obtenidos para el área bajo estudio, es factible realizar una centralización de las faenas de guardería para todo el patrimonio de la compañía, aplicando el modelo anteriormente descrito a la totalidad de las áreas de administración.

\section{REFERENCIAS}

(1) BODIN, L., B. GOLDEN, A. ASSAD y M. BALL. Routing and scheduling of vehicles and crews: The State of the Art, Computers and Operations Research. 1983, vol. 10, p. 63 - 211.

(2) LAWLER, E.L., J.K. LENSTRA, A.H.G. RINNOOY KAN, D.B. SHMOYS. The Traveling Salesman Problem: A guided tour of combinatorial optimization. Wiley series in Discrete Mathematics \& Optimization. 1985. New York, $476 \mathrm{p}$.

(3) DESROSIERS, J., Y. DUMAS, M.M. SOLOMON, F. SOUMIS. Time constrained routing and scheduling, In: M. BALL, T.L. MAGNANTI, C.L. MONNA, and G.L. NEMHAUSER, eds. Handbook in Operations Research and Management Science 8. Network Routing, NorthHolland, Amsterdam. 1995. p. 35-53. 
BOSQUE 26(2): 17-24, 2005

Optimización en la asignación de tareas en un sistema de guardería forestal

(4) CRAINIC, T.G., G. LAPORTE. Planning models for freight transportation, European Journal of Operational Research, 1997. vol. 97, p. 409-438.

(5) CHRISTOFIDES, N., A. MINGOZZI, P. TOTH. Exact algorithms for the vehicle routing problem, based on spanning tree and shortest path relaxations, Math. Prog. 1981. vol. 20, p. 255-282.

(6) CERDA, P. Problema de ruteo de vehículos: El caso de la empresa distribuidora Rabie S.A. Memoria de Título, para optar al título de Ingeniero Civil Industrial, Universidad de Concepción. 1999. Chile, 112 p.

(7) NUÑEZ, C. G. Revisión de algunos algoritmos exactos y diseño de una heurística para el problema de determinación de rutas óptimas. Informe de habilitación profe- sional para optar al título de Ingeniero Civil Industrial, Universidad de Concepción. 1995. Chile, 129 p.

(8) BERTSIMAS, D.J., G. VAN RYZIN. Stochastic and dynamic vehicle routing in the Euclidean plane with multiple capacitated vehicles. Operations Research. 1992. vol. 41 , p. 60-76.

(9) BRAMEL, J., E.G. COFFMAN, P.W. SHOR, D. SIMCHI-LEVI. Probabilistic analysis of the capacitated vehicle routing problem with unsplit demands. Operations Research. 1992. vol. 40, p. 1095-1106.

(10) AZOCAR, L. Optimización en las Operaciones en un Sistema de Guardería Forestal. Tesis de Magíster en Ingeniería Industrial, Universidad de Concepción. 2003. Chile, 89 p. 